\title{
Etnografía, Teoría Fundamentada y Frónesis: La Relevancia del Contexto
}

\author{
Ethnography, Grounded Theory and Phronesis: The Relevance of Context
}

Alexander Castleton'

\begin{abstract}
Resumo
En este artículo discutiré la relevancia del contexto para entender lo social. Sostengo que al reducir la realidad social a modelos estructurados, cerrados y predictivos, las posibilidades de comprender la vida humana se ven severamente restringidas. Baso esta idea en las nociones del sociólogo danés Bent Flybvjerg sobre el rol del término arsistotélico frónesis en la investigación sociológica. Trataré, además, de establecer cómo la frónesis es útil para enfoques cualitativos claves como la teoría fundamentada y la etnografía. Finalmente presentaré un ejemplo de trabajo empírico desarrollado dentro de dos fábricas de una empresa de productos lácteos en Uruguay (CASTLETON 2017).
\end{abstract}

Palavras chave: métodos cualitativos, etnografía, Teoría fundamentada

\begin{abstract}
In this article I will discuss the crucial relevance of context to make sense of the social. I claim that when reducing social reality to structured, closed, and predictive models, the possibilities of understanding human life are severely curtailed. To support this, I introduce and describe Danish sociologist Bent Flybvjerg's notions about the role of the Aristotelian term phronesis in sociological research, and I will especially try to establish how this idea is useful for key qualitative sociological approaches such as grounded theory and ethnography. I will finally present an example of empirical work developed within two factories of a dairy company in Uruguay (CASTLETON, 2017).
\end{abstract}

Keywords: qualitative methods, ethnography, Grounded Theory

\section{Introducción ${ }^{2}$}

Aristóteles, en su Ética a Nicómaco, define frónesis como "Una virtud intelectual que es razonada y capaz de actuar con respecto a cosas que son buenas o malas para el hombre" (apud FLYBVJERG, 2004, p.284). De acuerdo a esta definición, en este artículo seguiré al sociólogo danés Bent Flybvjerg en su afirmación de que la frónesis es tarea fundamental de la

\footnotetext{
1 Doutorando em Sociologia na Carleton University, Ottawa, Canadá. E-mail: alexandercastleton@cmail.carleton.ca

2 Las citas en inglés fueron traducidas al español por el autor.
} 
ciencia social, y mostraré que es una noción que tiene muchas similitudes con enfoques cualitativos tradicionales.

Una de las cruciales diferencias que otorga el enfoque fronético es que incentiva a que los investigadores pueden hacer un salto bastante audaz de lo descriptivo a lo normativo, y así abordar un problema no poco común en la investigación científica-social, a saber, la de responder preguntas del tipo ¿a quién o a qué afecta esta investigación? o ¿quién se da cuenta si estás equivocado en las investigaciones sociológicas? para las cuales la respuesta generalmente es nadie (FLYVBJERG, 2001). Intentaré articular esta forma de entender la sociología con otros enfoques que de manera similar proponen concentrarse en las experiencias vividas de los individuos, como son la sociología existencial (TIRYAKIAN,1962), el enfoque del cuidado (particularmente en su énfasis en atender situaciones concretas (PRESTON Y WICKSON, 2016; WICKSON ET. AL., 2017)), y la teoría del actor-red con sus nociones relacionadas a la dilución de las dicotomías agencia/estructura y macro/micro, y el principio de investigación que consiste en seguir a los propios actores (LAW, 1992; CALLON 1984; LATOUR 2005; CALLON Y LAW, 1997; RUMING, 2009). En este contexto, me centraré en dos métodos que pueden utilizarse particularmente en el espíritu de las ciencias sociales fronéticas: la etnografía y la teoría fundamentada. Pero primeramente, a continuación, presento el término frónesis de acuerdo a Flybvjerg.

\section{¿Qué es Frónesis?}

Frónesis es el término que Aristóteles usaba para referirse a la sabiduría práctica, la cual deriva de las experiencias de vivir en una comunidad particular. Está vinculado al buen juicio, la prudencia, y el sentido común. También es el término que Aristóteles sugirió para guiar la pólis; la persona que practica la frónesis, o el frónimos, sabe "cual es el fin u objetivo al que se dirige la buena vida" (TABACHNIK, 2013, p. 32). Frónesis no es algo que alguien posee, sino que se adquiere mediante prueba y error y mediante la imitación de frónimos preexistentes.

Frónesis se diferencia de los términos episteme y techné. Mientras que episteme se preocupa del conocimiento teórico y techné denota conocimiento 
técnico, frónesis enfatiza el conocimiento práctico y la ética práctica. Aristóteles fue explícito en su consideración de frónesis como la más importante de las tres virtudes intelectuales (epsiteme, techné y frónesis, ver tabla 1) porque es la actividad por la que la racionalidad instrumental es equilibrada por la racionalidad orientada a valores. Es más, el frónimos es el individuo que tiene conocimiento de cómo comportarse en cada situaciónconocimiento que nunca puede ser equiparado con, o ser reducido, a verdades generales y abstractas sobre situaciones sociales. En otras palabras, más que una clase de ciencia, frónesis es una habilidad tácita o sentido para hacer lo éticamente práctico, y de esta manera contrasta con el conocimiento universal y aproximaciones científicas deductivo-nomotéticas. $\mathrm{El}$ frónimos es el que mira las especificidades y los matices que componen cada situación concreta y quien, a través de su conocimiento práctico, es capaz de revelar su significado.

\section{Tabla 1}

Episteme: Conocimiento científico. Universal, invariable, independiente del contexto. Se basa sobre la racionalidad analitica general. El concepto original se conoce hoy en día con los términos 'epistemología' y epistémico'. Las pesquisas realizadas como episteme estarian focalizadas sobre el descubrir verdades y leyes universales.

Techné: Arte u oficio. Pragmático, variable, contexto-dependiente. Orientado a la producción. Basado sobre una racionalidad instrumental práctica que tiene un objetivo marcado y concreto. El concepto original aparece en términos como 'técnica', 'técnico' y 'tecnología'. Las investigaciones practicadas con techné serian planificadas de manera más eficiente a través de la racionalidad instrumental, donde 'más' se define en términos de valores y objetivos delineados por los que contratan las investigaciones, algunas veces negociados con los investigadores.

Frónesis: Ética. Deliberación sobre valores con referencia a la praxis. Pragmático, variable, dependiente de los contextos. Orientado hacia la acción. Basado en la practicidad y la racionalidad. El concepto original no se encontrará en ningún término contemporáneo análogo, ha desaparecido de la usanza moderna. La pesquisa y planificación practicada como frónesis se orientan hacia la deliberación sobre (e incluyendo) valores e intereses en la planificación. 
Frónesis, entonces, se basa en la experiencia específica contextual, en juicios de valor y en la práctica más que en la teoría abstracta. Se basa también en el diálogo, la narrativa y la exposición. Es por eso que el enfoque fronético incluye preguntas normativas más que meramente descriptivas, como ser: ¿Hacia dónde vamos? ¿Es deseable? ¿Qué se debe hacer? o ¿Quién gana y quién pierde? Se enfoca más en fines que en medios, y las respuestas que se buscan apuntan a aportar al diálogo social. Enfatiza la contingencia de la realidad, es decir, cómo las cosas podrían ser de otra manera.

Flyvbjerg (2001) aplicó estos principios a la sociología y desarrolló una aproximación a la investigación científica-social fronética. Desde este punto de vista, el autor danés rechaza la tendencia de las ciencias sociales a emular las ciencias naturales, algo que ha sido históricamente pernicioso debido a sus intrinsecas diferencias. Mientras que las ciencias sociales son buenas desarrollando análisis reflexivos que involucran la deliberación sobre valores, las ciencias naturales pretenden lograr teorias predictivas amplias y generales. De acuerdo a Flyvbjerg (2001), las ciencias sociales deben apuntar a la racionalidad valorativa y discutir lo que es deseable. Para este fin, en vez de buscar leyes sociales desarraigadas al estilo de las aproximaciones científico-naturales, es fundamental prestarle atención al contexto en el que se desarrolla la vida social. Flyvbjerg explica: El problema en el estudio de la actividad humana es que en cada
intento de búsqueda de una definición de una acción sin contexto
definido, es decir, una definición basada sobre reglas o leyes
abstractas, no necesariamente se está de acuerdo en la forma
pragmática en que una acción es definida por sus actores en un
situación social concreta. Los cientistas sociales no tienen una teoría
(reglas y leyes) sobre cómo los sujetos bajo estudio determinan lo que
constituye una acción, porque esa determinación deriva de
habilidades definidas por situaciones (dependientes de contextos
específicos) en las cuales los sujetos son expertos, y porque la teoría
por definición presupone independencia de un contexto
(FLYVBJERG, 2001, p.41).

Así entendida, la frónesis es muy similar a la fenomenología si entendemos a ésta como una hermenéutica de la cotidianidad (AAGAARD, 2018). La fenomenología busca desvelar aquello que pasa por desapercibido en el día a día, aquello que por estar tan cerca, no lo vemos. Es tratar de buscar cómo las estructuras se expresan en el diario acontecer y conforman 
a los actores. Aagaard (2018: 43) explica que "una buena descripción fenomenológica resuena con la vida y evoca el reconocimiento fenomenológico. El concepto de resonancia significa eco o reverberación. El objetivo de la fenomenología no es tanto el sorprender, sino tocar una cuerda de familiaridad con los lectores. Cuando un texto resuena con el lector, excita, estimula y pone a la persona en movimiento. Mueve al lector."

De esta manera, y con una fuerte impronta fenomenológica, Flyvbjerg presenta una forma novedosa de mirar las ciencias sociales que refiere a traer al frente significados y acciones contextuadas y la introduce como la forma más válida para realmente comprender la vida social. En la siguiente sección, reflexionaré sobre otras aproximaciones cualitativas al tema que mantienen este mismo espíritu y las articularé con una visión fronética.

\section{Frónesis, razón vital, y la sociología existencialista: la complejidad de la vida social y el rechazo del cientificismo}

El enfoque crucial sobre el contexto y el acercamiento cauto a los modelos predictivos es muy similar al rechazo del cientificismo compartido por muchos filósofos y científicos sociales. Por ejemplo, en forma notable, Nietzsche se manifestó en contra del positivismo en su periodo medio y final, mientras que Dilthey enfatizó que las ciencias sociales deben enfocarse en la verstehen, es decir, la comprensión por medio de la interpretación del comportamiento humano, ya que es precisamente esto lo que las diferencia de las ciencias naturales. Max Weber, más adelante, construiría su método sociológico sobre esa mismo noción. El filósofo español José Ortega y Gasset también se expresó sobre esta línea de pensamiento cuando hablaba de la "barbarie del especialismo" al referirse a los científicos de su tiempo como "sabios ignorantes", es decir, como cortos de vista imposibilitados de explicar la realidad fuera de su diminuta área de conocimiento. En forma similar a Ortega, el filósofo inglés contemporáneo Roger Scruton (2013, p. 38) sugirió que las maneras científico naturales y epistémicas de concebir la realidad humana no son apropiadas debido a que la condición humana se sostiene sobre la intersubjetividad y la responsabilidad recíproca (SCRUTON, 2017). 
Este filósofo también enfatizó la irreductibilidad de la subjetividad al análisis objetivo:

Como sujeto consciente, tengo una forma de mirar el mundo. E1 mundo aparece de cierta forma para mí, y este 'aparece' define mi perspectiva única. Todo ser consciente tiene una perspectiva parecida, y esto define lo que es ser más un sujeto que un objeto. Sin embargo, cuando hago un relato científico del mundo, describo exclusivamente objetos. Estoy describiendo las cosas como son, así como las leyes casuales y simples que las explican. Esta descripción no se da desde ninguna perspectiva en particular. No contiene palabras como "acá", "ahora" o "yo", y mientras su intención es explicar cómo parecen ser las cosas, lo hace formulando una teoría de cómo son. En suma, el sujeto es en principio inobservable para la ciencia - no porque exista en otro plano, sino porque no es parte del mundo empírico. Se ubica en la periferia de las cosas, como una especie de horizonte que nunca puede ser tomado "desde el otro lado", el lado de la subjetividad en sí misma (SCRUTON, 2017, p.38).

La idea que recalca aquí Scruton es la esencialidad de la perspectiva. La complejidad del contexto de la vida en el cual se mueven y actúan los seres humanos impide que se pueda capturar su esencia adecuadamente por medio de aproximaciones científico-naturales y predictivas. Esto resuena con lo que filósofos anteriores vinculados al existencialismo, como el ya mencionado Ortega y Gasset y su amigo y acólito más relevante, Julián Marias ${ }^{3}$, describieron como la metodología de la "razón vital". Con la "razón vital" Ortega desarrolló una filosofía que busca sobreponerse tanto al materialismo como al idealismo, como Marías explica:

La realidad radical es nuestra vida. La vida es lo que hacemos y lo que nos acontece. Vivir es lidiar con el mundo, dirigirse a él, actuando en él y cuidándolo. Por lo tanto las cosas no son anteriores, como creían los realistas, ni la subjetividad viene antes a las cosas como en la perspectiva de los idealistas. La realidad primaria y radical, donde el 'yo' y las cosas son solamente momentos abstractos, es en esencia la tarea dinámica que llamamos nuestra vida (MARÍAS, 1967, p.435).

Mientras que el materialismo asumía la existencia de una realidad objetiva, el idealismo afirmaba que la realidad era un producto de la mente humana proyectada hacia adentro. Con el método de la razón vital las cosas no pueden ser independientes del sujeto que los percibe-sujeto para quien la realidad externa tiene un significado particular. Pero tampoco puede ser puramente tema de auto-conciencia. La verdadera realidad radical es el de

\footnotetext{
${ }^{3}$ Aunque la etiqueta de existencialista para Ortega y Gasset ha sido discutida (ver WINECOFF,1970; MARÍAS, 1953).
} 
un sujeto (yo) y objeto (circunstancia) que son, en definitiva, inseparables y co-constituidos. La circunstancia no define la vida pero si la limita (ATENCIA PÁEZ, 2003). Simultáneamente, sin embargo, el 'yo' debe hacer su circunstancia. En virtud de su arrojamiento, se le fuerza a tomar decisiones y proyectarse hacia el futuro. La razón entonces se enraíza en la vida, en una biografia que la hace co-dependiente con la circunstancia. Esto es casi equivalente a decir que la razón es esencialmente histórica y que debe ser comprendida en forma biográfica y narrativa. Como Marias explica:

\begin{abstract}
La vida es, entonces, el órgano más propio a la comprensión. Es por eso que se puede decir que la razón es la vida humana. La realidad humana solamente puede ser comprendida en la vida referida a la totalidad en que está enraizada. Solamente cuando la vida en si misma funciona como razón es que podemos alcanzar algo humano. Esto, en resumidas cuentas, es lo que significa la razón vital (MARÍAS, 1967, p.440)
\end{abstract}

De ese modo, observando la realidad social como vidas situadas, como narrativas biográficas, nos aproximamos más claramente a su complejidad y a su condición única como experiencias vividas. Esto supone una crítica a lo que Aspers y Kohl (2013), influidos por el pos-humanismo y la socio-ontología de Heidegger, describieron como el "hechizo epistemológico-egológico" que ha sido reiteradamente objetado por sociólogos existencialistas. Estos se oponen a un "sociologismo" inerte que asfixia el aporte de la experiencia vital del individuo (TIRYAKIAN, 1962) y que ve al actor como una tabula rasa que simplemente internaliza las normas y los valores como un homo economicus (ADLER et al., 1987, p. 218). Altheide, por ejemplo, siguiendo esta línea de pensamiento, enfatiza la contingencia y la fluidez de la vida social—cualidades que la hacen irreductible:

Una ciencia social significativa debe ser capaz de dar sentido a lo que es importante para las personas reales, y no simplemente reducirlas a actores que "hacen" vida social. La sociología existencial busca articular la forma en que la acción se vuelve relevante en ciertos contextos. El conocimiento de los procedimientos invariantes no puede decirnos por qué un hombre mata y otro ama (ALTHEIDE, 1977, p. 148).

Andrea Fontana (1987, p. 3) también argumentó contra marcos teóricos abstractos, como el Parsoniano, afirmando que estos eliminan "la complejidad del comportamiento humano, dejándonos con una visión 
esquelética que de ninguna manera representa a los seres humanos que pueblan el mundo cotidiano". Entonces, la misión de este tipo de sociología es "estudiar a los seres humanos en su entorno natural — su diario vivir en la cotidianeidad de su mundo- y examinar la mayor parte de las complejas facetas de la experiencia humana" (FONTANA, 1987, p. 4). Las semejanzas aquí con la frónesis son evidentes, ya que, tanto los sociólogos existencialistas como Flyvbjerg demandan narraciones detalladas de las acciones humanas y de las estructuras situadas que son concomitantemente modificadas por la agencia de los individuos. Significa describir ricamente las circunstancias en las cuales el yo está sumergido — circunstancias que a su vez lo co-constituyen, dejando al mismo tiempo una puerta abierta para que la realidad pueda ser de otra manera.

\section{Vida, contexto y método: la relevancia de los estudios de caso}

Esta forma de entender las ciencias humanas enfocada en la complejidad y en la irreductibilidad de la vida - de tal forma que se diferencia radicalmente al cientificismo- lleva desde un punto de vista metodológico a la investigación de casos particulares. Prestando atención a casos concretos es que los matices y la ambigüedad de los contextos pueden ser apropiadamente valorados. La narrativa en si es la respuesta (FLYBVJERG, 2001). Lo que Flyvbjerg (2001) quiere decir es que la vida humana no se puede reducir solamente a seguir reglas ya que no hemos llegado a ninguna teoría social predictiva —además de que en las ciencias sociales no existen paradigmas como en las ciencias naturales. Entonces, al hacer investigación sociológica, debemos enfatizar más la comprensión que la predicción. Por esto, los estudios de caso y la narrativa son cruciales en tanto que el contexto en que está inmersa la gente no puede ser simplemente formalizado en teorías generales predictivas. De hecho, el contexto en el cual se lleva a cabo la acción social esta comprendido por las habilidades y conocimientos tácitos de los humanos, que están, a su vez, basados en juicios que no se pueden entender en términos de reglas formales. En otras palabras, no hay teoría alguna para el criterio humano en la medida que no 
puede ser reducido a fórmulas teóricas (FLYVBJERG, 2001.; cf. DREYFUS, 2014)

Recapitulando, frónesis es el énfasis en los valores, la práctica, el contexto y la normatividad. Se refiere a la discusión axiológica, es decir, a cuáles acciones son buenas o malas en un contexto social determinado. Para ello, los estudios de casos ayudan a desarrollar "una visión matizada de la realidad, incluyendo el hecho de que el comportamiento humano no puede ser cabalmente comprendido por reglas que gobiernan actos" (FLYVBJERG, 2006, p. 223). En consecuencia, los estudios de caso permiten focalizarse en situaciones de la vida real, examinando distintos puntos de vista mientras que las prácticas y usanzas sociales se desarrollan naturalmente, narrando historias en toda su diversidad y complejidad (FLYVBJERG, 2006, p.235238). Esto es útil, tal cual sugiere Flyvbjerg (2005), para que las ciencias sociales puedan producir análisis reflexivos sobre valores e intereses y contribuyan a las discusión de cómo estos afectan distintos grupos en una sociedad. Luego, el objetivo es que estas reflexiones alimenten el proceso de deliberación y toma de decisiones. De este modo, el objetivo de las ciencias sociales fronéticas es proveer material para el pensamiento y análisis, guiadas por problemas específicos más que por metodología (FLYVBJERG, 2005, p. 39-40).

Otro motivo por el cual la investigación debería intentar de proveer ejemplos concretos y narrativas detalladas de vida, es para sugerir cómo funciona el poder y cómo puede ser modificado para que fluya de otra manera. Esto debe ser tomando en cuenta que las repuestas definitivas o finales no están a nuestro alcance. No obstante, el objetivo debe ser producir materia prima para el diálogo y la práctica en cuestiones sociales (FLYVBJERG, 2005). Por lo tanto, este tipo de ciencia social debería elegir casos basándose en sus expectativas y su contenido de información, teniendo asimismo importancia estratégica en relación al problema de estudio en general. Debería, además, prestar atención a los casos que permitan eliminar prejuicios y preconceptos. De esta forma se gana en profundidad y riqueza pero se pierde en cobertura - cobertura poco fecunda 
a la cual estamos acostumbrados en la tradicional aproximación de las ciencias sociales (FLYVBJERG, 2005).

En resumen, la aproximación fronética de Flyvbjerg (2001, 2004, 2005, 2006) a las ciencias sociales implica los siguientes componentes principales: énfasis sobre situaciones particulares y contextos; basarse sobre las habilidades y mundo de la vida de la gente; hermenéutica; enfocarse en la perspectiva, el contexto desde un punto de vista histórico, y sobre relaciones de poder; análisis participativo; mantenerse cerca de la realidad tratando de describir en forma profunda, y percatándose de que 'tanto Dios como el diablo está en los detalles'; enfocarse en prácticas diarias y en el cómo (verstehen); producir narrativas que comienzan con lo concreto; analizar simultáneamente la relación estructura/agencia basándose en una aproximación orientada a producir diálogo social continuo en lugar de generar conocimiento unificado; perspectivismo, tratando de entender más que descubrir.

\section{Enfoque del cuidado feminista, la ciencias sociales como filosofía pública, y la teoría del actor-red como frónesis}

Flyvbjerg (2001) enfatiza las similitudes de su aproximación fronética a las presentadas en el libro de Bella et. al. Habits of the Heart (1985). La cercanía de ambos reside en su afirmación común de que las condiciones de fondo en el mundo social no son físicas, sino más bien patrones de comportamiento caracterizados por habilidades y experticias tácitas contingentes (cf. DREYFUS, 2014). Por lo tanto Flybvjerg (2001, 2004, 2005, 2006) y Bellah et. al (1985) sugieren que rara vez se puede predecir el mundo social de forma consistente por medio de variables únicas, ya que estas nunca son lo suficientemente independientes. Las variables pueden entenderse de forma precisa solamente en el contexto social, y por lo tanto el enfoque debe ser puesto en lo narrativo. Esto también implica tomar en cuenta el rol del analista en la medida que este no se mantiene en un vacío moral a-valorativo, sino que es en sí parte crucial del proceso narrativo. Para demostrar esto, Bellah et.al. (1985) toman su propia experiencia como ciudadanos de la muy compleja realidad que es los Estados Unidos, utilizando su exploración de la base del sentir común norteamericano como 
un insumo para deliberar. Los autores proponen una filosofia "pública" en la medida que por medio de su investigación pretenden involucrar al público en el diálogo. De este modo, contrastan métodos como las entrevistas en profundidad con encuestas, sugiriendo que estas, al resumir las opiniones de miles de encuestados, disminuyen la riqueza de la realidad social. Por lo tanto, Bellah et al. (1985) se vuelcan por las aproximaciones cualitativas que crean la posibilidad de conversaciones y discusiones públicas cuya cometido es, justamente, crear diálogo.

Bellah et.al. (1985) también remarcan el rol del investigador como una herramienta para establecer dicho diálogo. Esto es similar a aproximaciones feministas que se han concentrado en enfoques del cuidado más que en acercamientos consecuencialistas. La ética del cuidado feminista implica un énfasis en temas vinculados a "relaciones sociales comprendidas como cambiantes, dependencias, y distribuciones de poder, asi como a entender contextos específicos" (PRESTON Y WICKSON, 2016, p. 49). Preston y Wickson (2016) usan este marco para evaluar sistemas tecnológicos como los organismos genéticamente modificados (OGM), a los cuales conciben como agentes que cambian dramáticamente las relaciones sociales y que presentan importantes riesgos culturales y socio-económicos. De esta manera, sugieren el uso de una perspectiva feminista volcada a la ética del cuidado que incluya la comprensión de problemas sociales en términos de una ontología relacional que enfatiza el contexto y la particularidad. Esta perspectiva reconoce además el significado de la dependencia y el poder, e introduce así la preocupación sobre los más vulnerables enfatizando la dimensión afectiva de la ética y el rol importante de la narrativa. La ética feminista de los cuidados podría ser extrapolada del enfoque de Preston y Wickson $(2016,2017)$ sobre la tecnología como los OGM y aplicada a la investigación social en general, en cuanto a que propone algo que tiene más de un punto de contacto con la propuesta de Flyvbjerg (2001) de "hacer que las ciencias sociales importen". La ética del cuidado feminista y su concentración en los contextos, donde las posiciones parciales de los actores son considerados, y donde se incluye la dependencia de individuos corpóreos 
más que seres abstractos, transmite el mismo espíritu que las ciencias sociales fronéticas.

En virtud de las características de la perspectiva feminista, se puede establecer otro nexo entre el acercamiento fronético y la teoría del actor-red (ANT por su sigla en inglés). En realidad, la epistemología feminista debe mucho a estudios de ciencia y tecnología (conocidos como STS por su sigla en inglés) que fueron pioneros en el análisis de la ciencia como una práctica cultural (LATOUR Y WOOLGAR, 1997). También le debe en gran medida a los trabajos de autoras como Donna Haraway (1988) y sus propuestas del reconocimiento de las epistemologías situadas y del rol del investigador en la creación de conocimiento. En forma aún más radical, la ANT indica la dilución de la dualidad del sujeto/objeto hacia una simetría absoluta radicada entre actores humanos y no humanos, siendo ambas partes igualmente responsables en la creación de lo social (ver, por ejemplo, LATOUR, 2005). A través de su enfoque en la unión entre entidades como forma de conceptualizar el ordenamiento y reordenamiento de lo social, la ANT disuelve la separación entre micro y macro, enfatizando asimismo que las estructuras pueden ser cambiadas en la medida que la identidad de los actores, tanto humanos como no-humanos, sea (re)adaptada a una red en particular. John Law lo explica:

Es así que la teoría del actor-red analiza y desmitifica. Desmitifica
el poder de los poderosos. Dice que en última instancia no hay
diferencia, ninguna gran división, entre el poderoso y el
desgraciado. No hay tal cosa como la última instancia. Y como no
existe la última instancia como tal, en la práctica hay,
efectivamente, diferencias entre los poderosos y los desgraciados.
En efecto, diferencias entre los métodos y los materiales que
despliegan para generarse a si mismos. Nuestra labor es estudiar
esos materiales y esos métodos para entender como se auto
realizan, haciendo notar que podria, y muchas veces deberia, ser de
otra manera (LAW, 1992, p.390).

ANT, entonces, propone que la relación entre estructura y agencia no está grabada en piedra. Más bien, enfatiza lo fluido y lo dependiente de las asociaciones establecidas entre entidades múltiples que permanentemente componen y recomponen estructuras. La única manera de comprender estas asociaciones es a través de análisis cercanos a la realidad, contextuales y situados. Entonces, los autores de la ANT (como CALLON 1984; LATOUR, 
2005; CALLON Y LAW,1997) han introducido la idea de que la estrategia para analizar como se establecen los ordenes sociales consiste en 'seguir a los actores' permitiendo que ellos demuestren los límites del estudio. Autores como Law y Urry (2004) o Law $(1987,2004)$ han demostrado y remarcado repetidamente en este sentido que la realidad es compleja, caótica y heterogénea. Por eso, simultáneamente, debemos tener en cuenta las precauciones de Mol (2010, p.261-262) en cuanto a que ANT no es coherente y no persigue la predictibilidad - uno de los principios fundamentales de las ciencias sociales fronéticas. En palabras de Mol:

\begin{abstract}
ANT no es una teoria. No ofrece explicaciones causales ni método consistente, sino que toma más bien la forma de un repertorio. Si uno se conecta con ella se aprenden términos sensibilizadores, formas de preguntar, y técnicas para ver temas de adentro hacia afuera. Con estos, uno puede salir y recorrer nuevas sendas. Pero cuidado: nadie lo llevará de la mano, no hay seguridades... El punto no es insistir hasta que un patrón se afirme, sino de agregar cada vez más capas, y por ende, enriquecer su repertorio (MOL, 2010, p.261-262).
\end{abstract}

El análisis de ANT de las asociaciones, entonces, es apropiado para las ciencias sociales fronéticas dada su aproximación empírica y ontología relacional. Esta es la única manera para que los investigadores puedan desarrollar las narrativas que permitan identificar cómo estructuras, agencias, e identidades, son hechas y re-hechas en contextos determinados. También establece que es crucial mirar la agencia de no humanos en la cocreación de tales estructuras y agencias humanas, dando así voz a la realidad material que co-constituye la realidad social. De esta manera, la investigación, en su narrativa, puede seguir tanto valores como temas de poder, acercándose a la realidad, enfatizando pequeñas cosas, observando la práctica antes que el discurso, estudiando casos y contextos, juntando agencia y estructura, y, por último, entrando en diálogo con una polifonía de voces tanto humanas como no humanas (FLYVBJERG, 2001, p.130-140).

\title{
Fronésis, Etnografía y Teoría Fundamentada
}

Uno de los elementos clave de la ciencia social fronética es que es guiada por problemas concretos más que por metodologias. Esto significa que aproximaciones estructuradas a la realidad social deben ser 
reemplazadas por otras que fluyan más libremente y sean más flexibles. Prácticas tradicionales en sociología como la teoría fundamentada o la etnografía con sus técnicas como la observación participante o entrevistas en profundidad, son herramientas interesantes para desarrollar ciencia social fronética; especialmente para investigadores jóvenes en formación (como el que escribe este artículo) quienes no tienen ni el tiempo ni los recursos para sumergirse suficientemente en un escenario específico (en relación a este punto ver SUPERVIELLE Y ROBERTT, 2013). Ciertamente, las técnicas tradicionales del oficio pueden ser una guía para organizar el caos en que uno esta sumergido al analizar la construcción de la sociedad, especialmente con el seguimiento de los actores. Pero debemos mantener la advertencia de Flybvjerg en mente:

La investigación planeada fronéticamente no sigue un método, aunque las preguntas de método puedan ser relevantes. La investigación planeada fronéticamente está basada en problemas. Por lo tanto, tal investigación no puede ni debe suscribirse a priori a un método específico, como ser análisis de discurso, estadísticas, o métodos cualitativos, aunque cada uno o todos estos métodos puedan aparecer como relevantes en una investigación específica para enfrentar problemas concretos. Es imposible estar verdaderamente motivado por problemas específicos y simultáneamente estar comprometido con una metodologia en particular. Entonces, el tema primario que enfrenta la investigación planeada fronéticamente es arribar a un método investigativo que conteste efectivamente las cuatro preguntas de la racionalidad orientada a valores como una base para la acción. El método empleado para proveer tales respuestas es de importancia secundaria en el sentido que el método a utilizar será determinado, en definitiva, por los mismos problemas a analizar (FLYBVJERG, 2004, p. 291).

Es por eso que enfatizo la teoría fundamentada y la etnografía como herramientas más que como metodologías. Estas herramientas son útiles para comprender situaciones sociales, concretas y reales, su dinámica y los poderes en juego, además de que guían a los investigadores a focalizarse en contextos por inmersión en el campo, lo cual permite una perspectiva privilegiada de la realidad social.

Por un lado, la etnografia es el arte de describir un grupo humano inductivamente en su vida cotidiana. Consiste en intentar entender los fenómenos sociales desde el punto de vista de los sujetos del estudio, siendo conscientes que ellos mismos son los expertos (GÜBER, 2011). El objetivo es 
desenmarañar las estructuras conceptuales en las cuales las personas están inmersas y actúan, y de qué manera experimentan el mundo que las circunda y habitan. Comprende un ejercicio de descripción e interpretación a través del cual representar lo que los participantes piensan y dicen. Nunca es solamente una descripción de lo que las personas dicen ni como ven al mundo, como tampoco es una copia del mundo de los participantes. Es más bien una interpretación donde el investigador y los sujetos del estudio negocian sus reflexiones e ideas durante todo el proceso de investigación (GÜBER, 2011).

Por otro lado, la teoría fundamentada es una manera de construir teorias de corto alcance partiendo de observaciones del nivel más básico de las interacciones sociales. No se basa en teorias sociales pre-existentes (GLASER Y STRAUSS, 1967). En otras palabras, la teoria fundamentada permite que teorias emerjan inductivamente "del suelo" por medio de la creación de categorías provenientes de la información empírica recolectada, basada en la comparación constante de datos a medida que estos se generan. Para lograr esto, la teoría fundamentada sigue ciertos protocolos desarrollados por distintos autores como la de codificación de datos, análisis de casos negativos, la redacción de memorandos, etc. Sin embargo, hay que siempre tener en cuenta que estos procedimientos no garantizan la emergencia de una teoría satisfactoria. Charmaz (2006) afirma que el investigador interpreta datos más que "descubre" hechos o fenómenos.

Como un sociólogo joven, inspirado en la ciencia social fronética, paso a describir brevemente como esta puede ser aplicada retrospectivamente al que fue mi primer trabajo como investigador recién egresado de la universidad con un diploma en Sociología.

\section{Frónesis en el campo}

Después de recibir mi licenciatura en Sociología fui contratado para realizar trabajo de campo en un proyecto de investigación del Departamento de Sociología de la Universidad de la República de Uruguay. Consistía en un estudio de las relaciones laborales dentro de dos plantas de fabricación de 
productos lácteos en la mayor compañía del país. Mi rol era el de etnógrafo realizando observación participante. Esto implicó trabajar con el mismo estatus laboral que los trabajadores zafrales o eventuales, quienes eran contratados cuando había picos de producción. Fue, de hecho, un trabajo bastante extenuante, ya que además de trabajar efectivamente en la fábrica seis días a la semana, ocho horas al día, tenía que ejercitar constantemente mi memoria (ya que era imposible llevar una libreta de anotaciones al lugar de trabajo en las plantas) y dedicar varias horas al día al registro de mis observaciones luego de la jornada de trabajo. De esta experiencia, apliqué teoría fundamentada y publiqué un artículo (ver CASTLETON, 2017) donde presenté la figura siguiente:

Figura 1

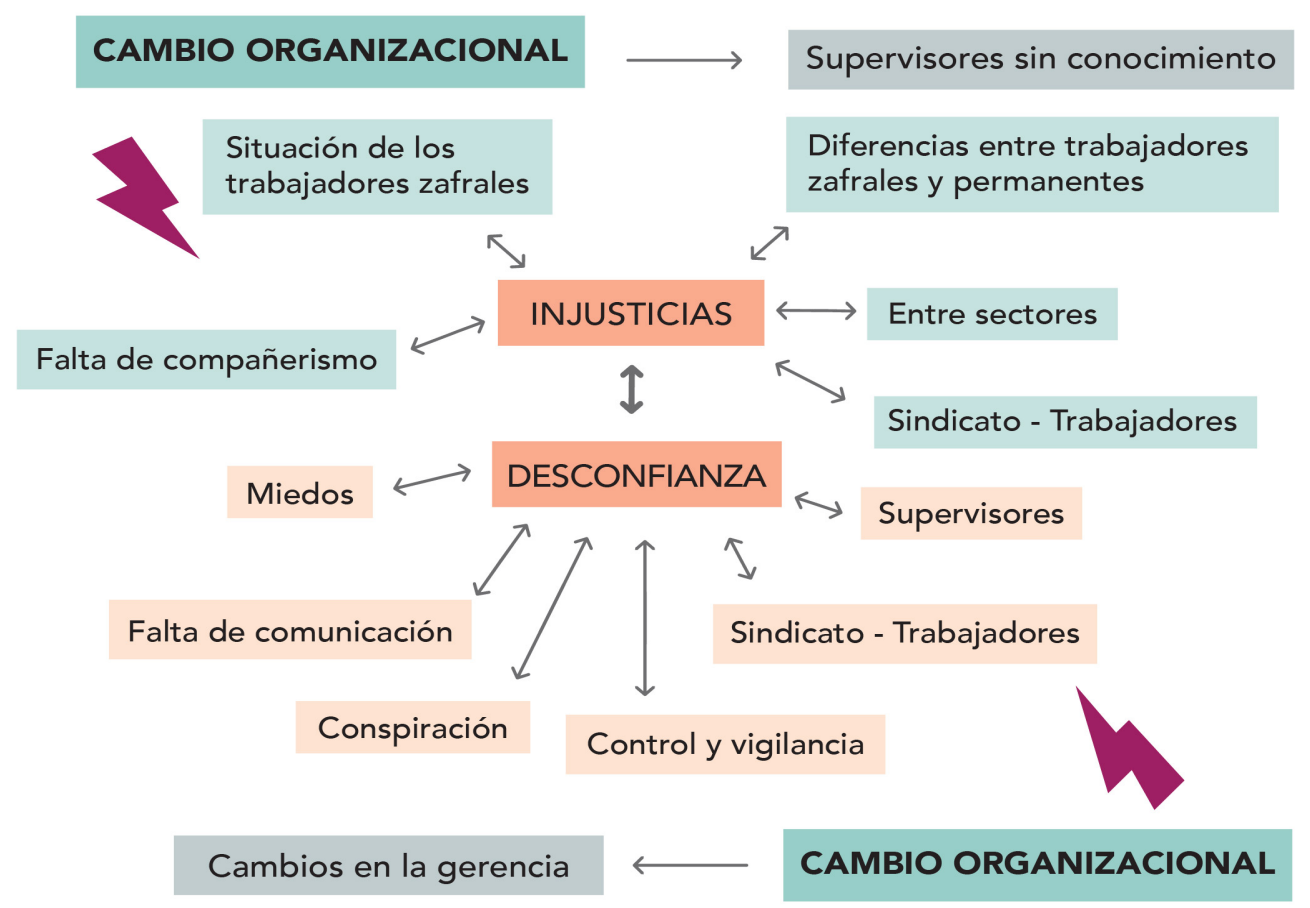

De esta figura, expliqué lo siguiente:

Aquí se muestra el concepto de cambio organizacional como el marco en el que las injusticias y la desconfianza surgieron dentro de la fábrica. De este modo, el cambio organizativo en la fábrica uruguaya se expresa concretamente en las modificaciones de la gestión. Los relámpagos se refieren a una transformación que golpea desde arriba, externamente, a la realidad interna de la fábrica y que acarreó las situaciones mencionadas. Por una parte, había una 
influencia bidireccional entre los sentimientos de injusticia, la situación de los trabajadores temporales, la falta de asociación, la distinción entre los empleados permanentes y temporales, la diferencia entre los sectores y la relación entre el sindicato y los trabajadores. Por otro, existía una relación bidireccional entre ciertos rasgos, como el temor, la falta de comunicación, los sentimientos de conspiración, el control, la vigilancia y la relación entre el sindicato, los empleados y los supervisores (CASTLETON, 2017, p.7).

Creo que esta investigación suscribe a la lógica de las ciencias sociales fronéticas porque toca varios de sus principios más importantes, como ser las experiencias contextuales específicas, la aplicación de juicios de valor y la práctica más que la teoría. Fue también un trabajo dialógico basado en narrativa y exposición. En este sentido, era muy difícil intentar llegar a una solución concreta o una especie de tratamiento específico a los problemas como quería la directiva de la compañia - sino que se buscó contribuir al dialogo social en fábricas con serios problemas de relacionamiento entre directiva, mandos medios, y trabajadores. Para empezar, este trabajo está basado sobre el contexto de la fábrica - un contexto donde había distintos factores de poder en juego. De ese contexto expuse una dinámica social que se produjo en el campo. Produje una narrativa que mostraba cómo las macro circunstancias de la situación económica regional tenían profundos efectos sobre las micro relaciones en un contexto específico. De alguna manera, pude mostrar la degradación de ambas, focalizándome sobre el impacto que las circunstancias cambiantes tenían sobre las emociones de las personas, y cómo relaciones sociales largamente establecidas eran erosionadas por cambios regionales exógenos a la organización, y por nuevas dinámicas de poder.

En el contexto de las fábricas, distintos agentes no-humanos como ser normativas, máquinas, roles e ideologías, configuraban las identidades de las personas. Por ejemplo, la inestabilidad propia del trabajador zafral estaba constituida por una serie de prácticas que nos definían, como ser el tener que recurrir a elementos como una escoba, un balde o un lampazo, para rápidamente simular que estábamos ocupados cuando había algún problema con las máquinas o alguna línea de producción. Cuando esto sucedía, los trabajadores efectivos (contratados sin término) simplemente se 
relajaban o no tenían la presión que nosotros, los zafrales o eventuales, teníamos. La asociación del trabajador zafral o eventual con una escoba, por ejemplo, significaba que tenía el ímpetu propio del buen trabajador, y de esa manera quería que el supervisor quedara bien impresionado, y le diera una buena calificación. (Los trabajadores zafrales o eventuales eran calificados en una serie de ítems y al final del contrato, usualmente de seis meses, se les daba la calificación, que tenía un gran impacto en las posibilidades de ser contratados por otros seis meses, o, idealmente, sin término).

No es mi intención reproducir aquí el artículo donde describo profundamente la situación en las fábricas. Pero esta presión a la que estaban sometidos los zafrales, que usualmente era acompañada por un sentimiento de abandono por parte del sindicato, es tan solo un ejemplo de un síntoma de la transformación que una empresa que en el pasado era considerada por sus trabajadores como "la casa", se había convertido en un espacio alienante e inherentemente conflictivo. Si lo entendemos como filosofia pública, sin dudas este trabajo contribuye a entender los social en su verdadera complejidad y apunta a ser insumo para la discusión y la deliberación, abrazando así la manera fronética de hacer ciencias sociales una manera que establece la suprema relevancia del contexto para entender las estructuras, agencias, y relaciones de poder en las cuales lo social se hace y se re-hace constantemente.

\section{Conclusión}

En este artículo me enfoqué en el concepto de contexto como fundamental para entender de forma debida la complejidad y ambigüedad de la realidad social. Utilicé los conceptos de Bent Flyvbjerg (2001, 2004, 2005, 2006) de la frónesis como una guía útil a las ciencias sociales. Además, presenté, articulé, y discutí la relevancia de la sociología existencial, la ética del cuidado, las ciencias sociales como filosofia pública, y la teoría de actorred como herramientas importantes para entender las cotidianeidad de la vida social, o en otras palabras, la recursividad plantada entre el individuo en sociedad y la sociedad en el individuo. 
Frónesis, entonces, se refiere a la sabiduria práctica que permite que el frónimos, es decir, el individuo que la posee, tenga una comprensión sutil de lo que realmente importa y está en juego en los procesos sociales. Esto no se hace a través de modelos complejos, sino más bien por la minuciosa descripción de contextos sociales, y también encierra una visión normativa más que una visión meramente descriptiva, a través de la cual el investigador señala relaciones de poder desiguales que tienen impactos profundos en las emociones de los individuos.

Propongo que este tipo de pesquisa cualitativa es crucial a los efectos de entender la complejidad de la vida social - una complejidad que no puede ser condensada en modelos estructurados.

\section{REFERÊNCIAS}

AAGAARD, Jesper. Striving for experiential resonance: Critique, postcritique and phenomenology. Qualitative Studies, vol.1, n.37, 2018.

ADLER, Patricia; ADLER, Peter \& FONTANA, Andrea. Everyday life sociology. Annual Review of Sociology, vol.13, n.1, 1987.

ALTHEIDE, David. The sociology of Alfred Schutz. In: DOUGLAS, John; JOHNSON, John. Existential Sociology. Cambridge: Cambridge University Press, 1977.

ASPERS, Patrik; KOHL, Sebastian. Heidegger and socio-ontology: A sociological reading. Journal of Classical Sociology, vol.13, n.4, 2013.

ATENCIA PÁEZ, José María. Ortega y Gasset, meditador de la técnica. Argumentos de Razón Técnica, n.6, 2003.

BELLAH, Robert; SULLIVAN, William; SWIDLER, Ann; TIPTON, Steven. Habits of the Heart: Individualism and Commitment in American Life. Berkeley: University of California Press, 1985.

CALLON, Michael. Some elements of a sociology of translation: domestication of the scallops and the fishermen of St Brieuc Bay. The Sociological Review, n.32, 1984.

CALLON, Michael; LAW, John. After the individual in society: Lessons on collectivity from science, technology and society. Canadian Journal of Sociology/Cahiers Canadiens de Sociologie, n.22, 1997. 
CASTLETON, Alexander. Reestructura y cambio social en "la casa": el caso de una fábrica uruguaya. Revista Latinoamericana de Antropologia del Trabajo, vol.1, n.1, 2017.

CHARMAZ, Cathy. Constructing Grounded Theory: a Practical Guide Through Qualitative Theory. London: Sage Publications, 2006.

DREYFUS, Hubert. Skillful Coping: Essays on the phenomenology of everyday perception and action. Oxford: Oxford University Press, 2014.

FONTANA, Andrea. Introduction: Existential Sociology and the Self. In: KOTARBA, Joseph A.; FONTANA, Andrea. The Existential Self in Society. Chicago: University of Chicago Press, 1987.

FLYVBJERG, Bent. Phronetic planning research: Theoretical and methodological reflections. Planning Theory \& Practice, vol.5, n.3, 2004.

Making social science matter: Why social inquiry fails and how it can succeed again. Cambridge: Cambridge University Press, 2001.

. Five misunderstandings about case-study research. Qualitative inquiry, vol.12, n.2, 2006.

Social science that matters. Foresight Europe, n.2, 2005.

GLASER, Barney; STRAUSS, Anselm. Discovery of grounded theory: Strategies for qualitative research. Chicago: Aldine, 1967.

GÜBER, Rosanna. La Etnografia: Método, campo y reflexividad. Buenos Aires: Siglo Veintiuno Editores, 2011.

HARAWAY, Donna. Situated knowledges: The science question in feminism and the privilege of partial perspective. Feminist studies, vol.14, n.3, 1988.

LATOUR, Bruno. Reassembling the Social: An Introduction to ActorNetwork Theory. Oxford: Oxford University Press, 2005.

LATOUR, Bruno; WOOLGAR, Steve. Laboratory Life: The Social Constructionof Scientific Facts. London: Sage, 1977.

LAW, John. After method: Mess in social science research. Routledge: New York, 2004.

Notes on the theory of the actor-network: ordering, strategy, and heterogeneity. Systems practice, vol.5, n.4, 1992.

Technology, Closure and Heterogeneous Engineering: The Case of the Portuguese Expansion. In: BIJKER, Wiebe; PINCH, Trevor; HUGHES, Thomas P. The Social Construction of Technological Systems. Cambridge: MIT Press, 1987. 
LAW, John; URRY, John. Enacting the social. Economy and society, vol.33, n.3, 2004.

MARIAS, Julian. History of Philosophy. Dover Publications: New York. 1967

MARIAS, Julian. E1 Existencialismo en España. Bogotá: Ediciones Universidad Nacional de Colombia, 1953.

MOL, Anne Marie. Actor-network theory: sensitive terms and enduring tensions. Kölner Zeitschrift für Soziologie und Sozialpsychologie. Sonderheft, n.50, 2010.

PRESTON, Christopher; WICKSON, Fern. Broadening the lens for the governance of emerging technologies: Care ethics and agricultural biotechnology. Technology in Society, n.45, 2016.

RUMING, Kristian. Following the Actors: mobilizing an actor-network theory methodology in geography. Australian Geographer, vol.40, n.4, 2009.

SCRUTON, Roger. On Human Nature. New Jersey: Princeton, 2017.

Scientism in the Arts and Humanities. The New Atlantis, 2013. Disponivel em: https://www.thenewatlantis.com/publications/scientism-inthe-arts-and-humanities. Acesso em: 12/08/2018.

SUPERVIELLE, Marcos; ROBERTT, Pedro. Observación etnográfica en un contexto industrial. Aplicación práctica de algunos principios de investigación. Revista Latinoamericana de Metodologia de la Investigación Social, n.5, 2013.

TABACHNICK, David. The Great Reversal: How We Let Technology Take Control of the Planet. Toronto: University of Toronto Press, 2013.

TIRYAKIAN, Edward. Existentialism and Sociologism. Englewood Cliffs: Prentice-Hall, 1962.

WICKSON, Fern; PRESTON, Christopher; BINIMELIS, Rosa; HERRERO, Amaranta; HARTLEY, Sarah; WYNBERG, Rachel \& WYNNE, Brian. Addressing Socio-Economic and Ethical Considerations in Biotechnology Governance: The Potential of a New Politics of Care. Food Ethics, vol.1, n.2, 2017.

WINECOFF DIAZ, Janet. The Major Themes of Existentialism in the Works of José Ortega y Gasset. Chapell Hill: University of North Carolina Press, 1970. 